\title{
Article \\ Solvent-Free Process for the Development of Photocatalytic Membranes
}

\author{
Rosa M. Huertas ${ }^{1,2}$, Maria C. Fraga ${ }^{1,2}$, João G. Crespo ${ }^{2}$ and Vanessa J. Pereira ${ }^{1,3, *}$ \\ 1 iBET, Instituto de Biologia Experimental e Tecnológica, Apartado 12, 2780-901 Oeiras, Portugal; \\ rosa.huertas@ibet.pt (R.M.H.); mariadocarmo.fraga@gmail.com (M.C.F.) \\ 2 LAQV-REQUIMTE, Departamento de Química, Faculdade de Ciências e Tecnologia, \\ Universidade Nova de Lisboa (UNL), 2829-516 Caparica, Portugal; jgc@fct.unl.pt \\ 3 Instituto de Tecnologia Química e Biológica António Xavier, Universidade Nova de Lisboa, \\ 2784 Oeiras, Portugal \\ * Correspondence: vanessap@ibet.pt; Tel.: +351-214-469-554
}

Received: 8 October 2019; Accepted: 25 November 2019; Published: 6 December 2019

\begin{abstract}
This work described a new sustainable method for the fabrication of ceramic membranes with high photocatalytic activity, through a simple sol-gel route. The photocatalytic surfaces, prepared at low temperature and under solvent-free conditions, exhibited a narrow pore size distribution and homogeneity without cracks. These surfaces have shown a highly efficient and reproducible behavior for the degradation of methylene blue. Given their characterization results, the microfiltration photocatalytic membranes produced in this study using solvent-free conditions are expected to effectively retain microorganisms, such as bacteria and fungi that could then be inactivated by photocatalysis.
\end{abstract}

Keywords: photocatalytic membranes; solvent-free sol-gel modification; temperature effect; membrane morphology; photocatalytic performance

\section{Introduction}

Silicon carbide membranes have been reported as effective supports that can be easily functionalized to provide materials with catalytic activity [1,2]. Moreover, due to its chemical and thermal resistance as well as mechanical properties, they are useful for harsh environmental and sustainable process applications [3-5]. Previous works demonstrated that silicon carbide membranes have a smooth top layer, controlled porosity, high permeability, and proved to be efficient in removing total suspended solids, as well as oil and grease when used to treat oily wastewaters $[4,6]$. The main problem associated with the use of membranes is fouling formation that can decrease the flux and the lifetime of the membrane.

Photocatalytic membrane reactors that combine filtration and degradation/inactivation in the same compartment have been described to have great potential for use in water and wastewater treatment [7]. Combining advanced oxidation processes with membrane filtration can overcome fouling problems and increase the effectiveness of the treatment process. The modification of membranes can also be used to decrease their molecular weight cut-off by reducing the pore size.

Titanium dioxide $\left(\mathrm{TiO}_{2}\right)$ is the most commonly used material for the fabrication of photocatalytic membranes due to its high reactivity, chemical and thermal stability, low cost, reusability, and high photocatalytic performance [7-9].

Sol-gel has been widely reported as effective for the synthesis of $\mathrm{TiO}_{2}$-based materials $[10,11]$. This technique can be used to modify different supports, such as membranes, to produce photocatalytic membranes, control their porosity [12-15], and eliminate surface defects. 
The combination of silicon dioxide $\left(\mathrm{SiO}_{2}\right)$ and $\mathrm{TiO}_{2}$ has been reported to improve the photocatalytic activity of $\mathrm{TiO}_{2}$ [16-18].

In previous work, a sol-gel process using ethanol as a solvent was used to coat $\mathrm{SiC}$ membranes with silicon dioxide $\left(\mathrm{SiO}_{2}\right)$ and $\mathrm{TiO}_{2}$ Degussa nanoparticles [19]. As a result, reusable photocatalytic membranes were obtained with a lower molecular weight cut-off, as well as higher hydrophilicity and oleophilicity, compared to the unmodified membranes. Assays performed in a dead-end filtration system combined with UV light confirmed their high potential to degrade organic contaminants [19].

Extremely high removals of total suspended solids, chemical oxygen demand, total organic carbon, phenolic and volatile compounds were obtained when these hybrid photocatalytic membranes were tested in a new hybrid photocatalytic membrane reactor to treat olive mill wastewaters [20].

Since the sol-gel procedure employs a high volume of solvent to modify large membrane areas needed at industrial scale, the development of solvent-free modification methods directly bound up with the principle of green chemistry [21], and would therefore highly increase the sustainability besides decreasing the membrane production costs.

The aim of the present work was to validate a new low cost and environmentally friendly methodology - based on the use of aqueous solutions-to produce $\mathrm{SiC}$ membranes with high photocatalytic activity.

\section{Results and Discussion}

\subsection{Photocatalytic Performance}

The total percent removal (photocatalytic activity, including adsorption obtained after $60 \mathrm{~min}$ exposure to UV light) and the adsorption of methylene blue obtained for the different membranes are shown in Figure 1 and Table 1. The removal of methylene blue was also measured in the absence of the photocatalyst (without membranes) under the same experimental conditions (Figure 1). A single experiment was conducted to test the photocatalytic activity of the membranes modified using solvent. In these experiments, the error was extremely low because it corresponded to four measurements of methylene blue concentration obtained after each photocatalytic experiment. The higher errors observed for the other samples (methylene blue, control, and solvent-free membranes) could be explained since they were calculated taking into account two duplicate experiments. Two duplicate experiments were conducted to test the photodegradation of methylene blue, as well as the photocatalytic performance of the unmodified and modified membranes (using two different pieces of the membrane).

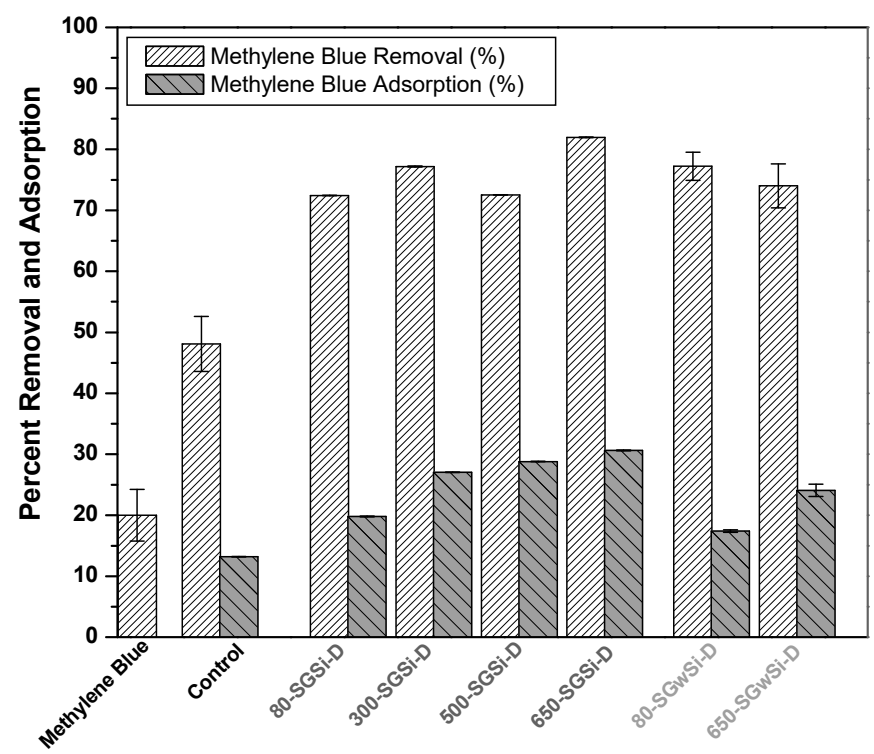

Figure 1. Comparison of the removal of methylene blue after $60 \mathrm{~min}$ (direct photolysis) with removal and adsorption of methylene blue using the control and the modified membranes. 
Table 1. Percent removal and adsorption measured using the unmodified (control) and modified membranes (60 $\mathrm{min})$.

\begin{tabular}{ccc}
\hline Membranes & $\begin{array}{c}\text { Methylene Blue } \\
\text { Removal (\%) }\end{array}$ & $\begin{array}{c}\text { Methylene Blue } \\
\text { Adsorption (\%) }\end{array}$ \\
\hline Control & 48 & 13 \\
\hline 80-SGSi-D & 72 & 20 \\
\hline 300-SGSi-D & 77 & 27 \\
\hline 500-SGSi-D & 73 & 29 \\
\hline 650-SGSi-D & 82 & 31 \\
\hline 80-SGwSi-D & 77 & 17 \\
\hline 650-SGwSi-D & 74 & 24 \\
\hline
\end{tabular}

The increase in the direct photolysis results obtained in these experiments (Figure 1, methylene blue column) compared to previous work [19] was due to a different medium pressure ultraviolet lamp used in this work. The removal due to direct photolysis of methylene blue (without membrane) was around $20 \%$, whereas when the control membrane was tested, an important self-photoactivity was observed, which was expected taking into account the results obtained in previous work [19], achieving a percent removal of $48 \%$.

The modified membranes obtained were identified as T-SGwSi-D and T-SGwSi-D, where T denotes the temperature applied over modified substrates samples, and the letter $\mathrm{w}$ differentiates the samples prepared using water as a matrix instead of solvent in the sol-gel process. Table 1 shows that the increase in the percent removals obtained by adding the photocatalytic layers was between $24 \%$ and $34 \%$.

The total removal obtained for modified membranes using solvent during the sol-gel process and subjected to different temperatures 80-SGSi-D, 300-SGSi-D, and 500-SGSi-D was around $76 \%$ that proved to be 1.5-1.6 times more photocatalytically active than the control membrane. Even though the membrane 650-SGSi-D subjected to the highest temperature during the thermal protocol achieved the highest total removal of $82 \%$ (Figure 1; Table 1), the differences in the photocatalytic behavior of this and the other thermally-treated membranes were not notorious (10\% difference between the removal efficiency of the membrane subject to $80^{\circ} \mathrm{C}$ and $650{ }^{\circ} \mathrm{C}$ ). This behavior could be due to the similarities among properties that influence the photocatalytic activity, such as surface area, number of active sites for radical formation, pore size distribution, or pore accessibility.

The solvent-free modified photocatalytic membranes were, therefore, prepared using the lowest temperature $\left(80^{\circ} \mathrm{C}\right)$, which would lead to reduced costs in terms of energy consumption, as well as the highest temperature $\left(650^{\circ} \mathrm{C}\right)$, which achieved the best performance when the membranes were modified with solvent. Distilled water was used as an aqueous solution for the sol-gel process to produce the T-SGwSi-D membranes. The membranes obtained were named 80-SGwSi-D and 650-SGwSi-D.

Figure 1 shows that the 80-SGwSi-D membrane achieved higher total removal and lower adsorption (Table 1) than the 650-SGwSi-D membrane and, therefore, had the highest photocatalytic effectiveness (1.6 times better than the control membrane).

Pseudo-first-order decay kinetics was observed. The time-based degradation rate constant $\left(\mathrm{k}_{\mathrm{t}}\right)$ was obtained from the slope of the relation between $\ln \left(C_{t} / C_{0}\right)$ and time (Equation (1)), where $C_{0}$ and $C_{t}$ correspond to the concentration of the dye measured before and after different $\mathrm{UV}$ exposures times.

$$
\ln \left(\frac{C_{t}}{C_{0}}\right)=-\mathrm{k} \cdot \mathrm{t}
$$


Table 2 and Figure S1 present the time-based degradation rate constants and the half-life times determined. The degradation rate constants and Equation (1) could be used to predict how the degradation of methylene blue is expected to change as a function of the exposure time.

Table 2. Calculated degradation rate constants $(\mathrm{k})$ and half-life time $\left(\mathrm{t}_{1 / 2}\right)$ values.

\begin{tabular}{ccc}
\hline Membranes & $\mathbf{k}\left(\mathbf{m i n}^{-\mathbf{1}}\right)$ & $\mathbf{t}_{\mathbf{1} / \mathbf{2}}(\mathbf{m i n})$ \\
\hline Control & 0.0110 & 63 \\
\hline Methylene blue & 0.0040 & 192 \\
\hline 80-SGSi-D & 0.0220 & 31 \\
\hline 300-SGSi-D & 0.0244 & 28 \\
\hline 500-SGSi-D & 0.0216 & 32 \\
\hline 650-SGSi-D & 0.0285 & 24 \\
\hline 80-SGwSi-D & 0.0275 & 25 \\
\hline 650-SGwSi-D & 0.0225 & 31 \\
\hline
\end{tabular}

Assessment of the Reusability Potential of the Membranes

One of the pre-requisites for using the developed photocatalytic membranes at the industrial level is to investigate the long-term stability and reusability of these materials. Thus, the photocatalytic activity of the membranes prepared (80-SGwSi-D and 650-SGwSi-D) was evaluated and compared after five consecutive degradation experiments using two different batches of silicon carbide membranes (labeled as Membrane I and Membrane II), prepared using the same chemical and thermal protocols. The results obtained under the same experimental conditions are depicted in Figure 2. The error was calculated based on four measurements of methylene blue concentration obtained after each photocatalytic experiment.

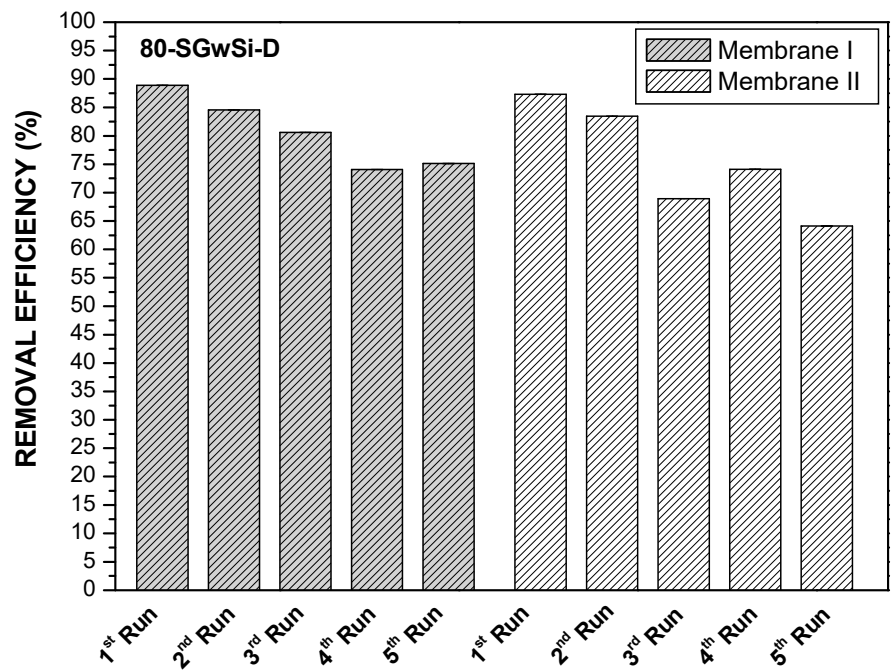

Figure 2. Cont. 


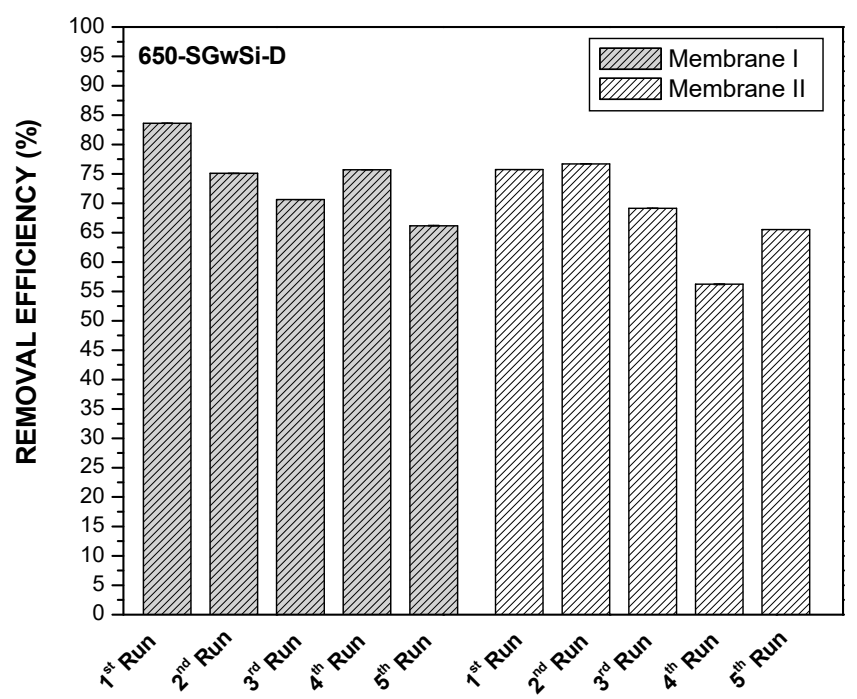

Figure 2. Removal efficiency obtained after $60 \mathrm{~min}$ for five consecutive photocatalytic experiments using two different membrane batches for the solvent-free 80-SGwSi-D (top) and 650-SGwSi-D (bottom) modified membranes.

When comparing the membranes (T-SGSi-D) prepared by the deposition of $\mathrm{SiO}_{2}-\mathrm{TiO}_{2}$ using a solvent (Figure 1) with the solvent-free process (T-SGwSi-D), we could observe that the photocatalytic activity of the solvent-free process achieved higher removal values (up to $89 \%$ for the membrane 80-SGwSi-D; Figure 2). Comparing the efficiency of the modified membranes, we could observe that the photocatalytic activity of the $80-\mathrm{SGwSi}$-D membrane was roughly maintained after five repeated runs with an average total removal of $81 \% \pm 6$ and $76 \% \pm 10$ for Membrane I and Membrane II, respectively (Figure 2), whereas the membrane 650-SGwSi-D showed lower photocatalytic effectiveness and/or higher variability (with an average total degradation of $74 \% \pm 11$ and $69 \% \pm 11$ obtained for Membrane I and Membrane II, respectively). Similar average total degradation values were obtained using the two thermal protocols tested. These results showed that using a lower temperature in the final thermal protocol was enough to induce photocatalytic activity, which would lead to energy savings.

A final long term assay was conducted using the solvent-free membranes. Removal below the detection limits of methylene blue was achieved after $240 \mathrm{~min}$ of UV exposure for the 650-SGwSi-D membrane and after $160 \mathrm{~min}$ for $80-\mathrm{SGwSi}-\mathrm{D}$ being the latter the most efficient membrane (Figure 3). Error bars correspond to duplicate experiments.

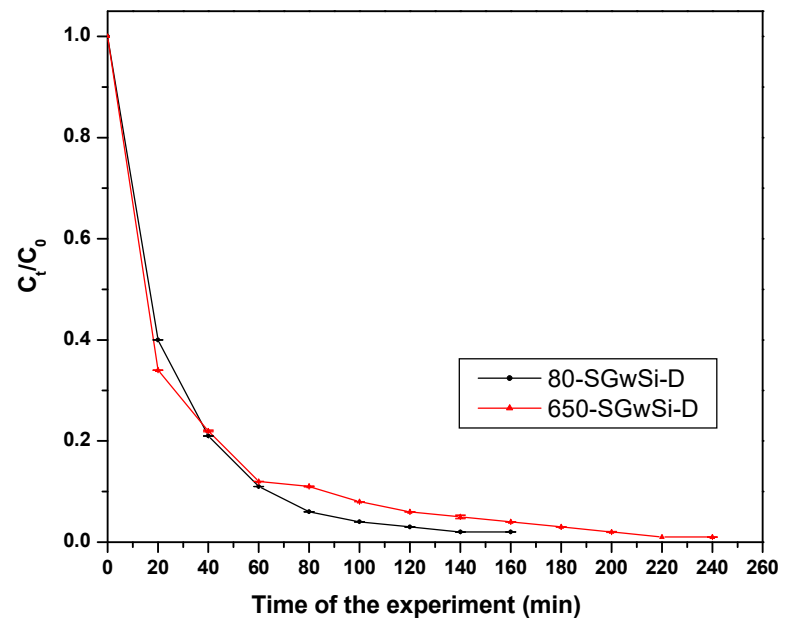

Figure 3. Total removal photocatalytic experiment conducted with the solvent-free membranes 80-SGwSi-D and 650-SGwSi-D. 
Comparing the efficiency of the membrane 80-SGwSi-D (Membrane I) with the control membrane, we could observe a stabilization of the photocatalytic performance around $75 \%$, maintaining a good performance after repeated six runs. On the contrary, the photo corrosion of the silicon carbide material [22] of the control membrane could induce a high decrease in the removal performance observed after three runs, achieving a removal efficiency up to $24 \%$ in the third run, which was extremely similar to the removal of methylene blue by direct photolysis (Figure 4).

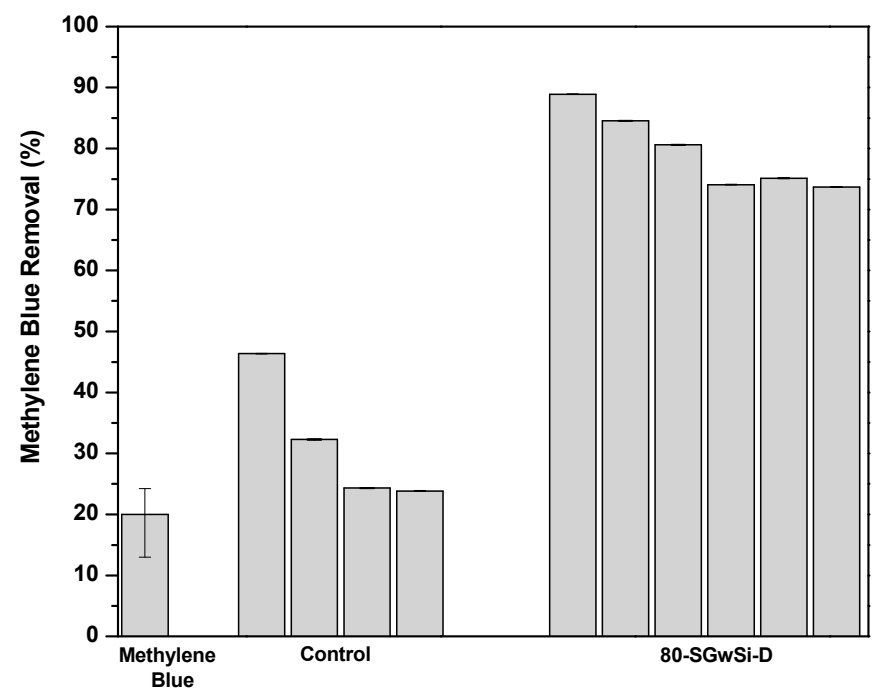

Figure 4. Comparison of the degradation of methylene blue by direct photolysis with the removal of methylene blue using the unmodified substrates (control) and solvent-free 80-SGSi-D modified membrane (indirect photolysis).

In these consecutive experiments, the error obtained for the control and the modified membrane was extremely low because it corresponded to four measurements of methylene blue concentration obtained after each photocatalytic experiment. The higher errors observed for the methylene blue direct photolysis could be explained since they were calculated taking, into account, two duplicate experiments.

The results obtained proved the positive effect of using water as a matrix instead of using ethanol during the sol-gel process, as well as using lower temperature values for achieving high photocatalytic performance, leading to a more sustainable production process (in terms of solvent consumption and energy costs).

\subsection{Morphology Characterization}

The morphology and homogeneity of the top layer and cross-section of the unmodified (control) and modified membranes using the solvent-free method were observed by SEM (Figure 5).

Figure 5 shows that the top layer of the control membrane presented an irregular surface with variable thickness. The 80-SGwSi-D and 650-SGwSi-D membranes exhibited a high nanoporous structure with a few surface irregularities due to the grains of titanium dioxide [19] and regular thickness. The 80-SGwSi-D membrane has a more uniform surface compared to the 650-SGwSi-D membrane that presented micro-cracks probably as a consequence of shrinkage during the final thermal protocol applied [23].

The cross-section images of the substrates presented in Figure 5 for the modified membrane showed that there was also no infiltration of the sol-gel solution through the membranes, showing a thickness with an average of $6.86 \pm 1.04 \mu \mathrm{m}$ for the 80-SGwSi-D and a reduction in the thickness of $5.60 \pm 1.00 \mu \mathrm{m}$ for the $650-\mathrm{SGwSi}-\mathrm{D}$ membrane, which could be explained due to the thermal densification of the film [24]. 


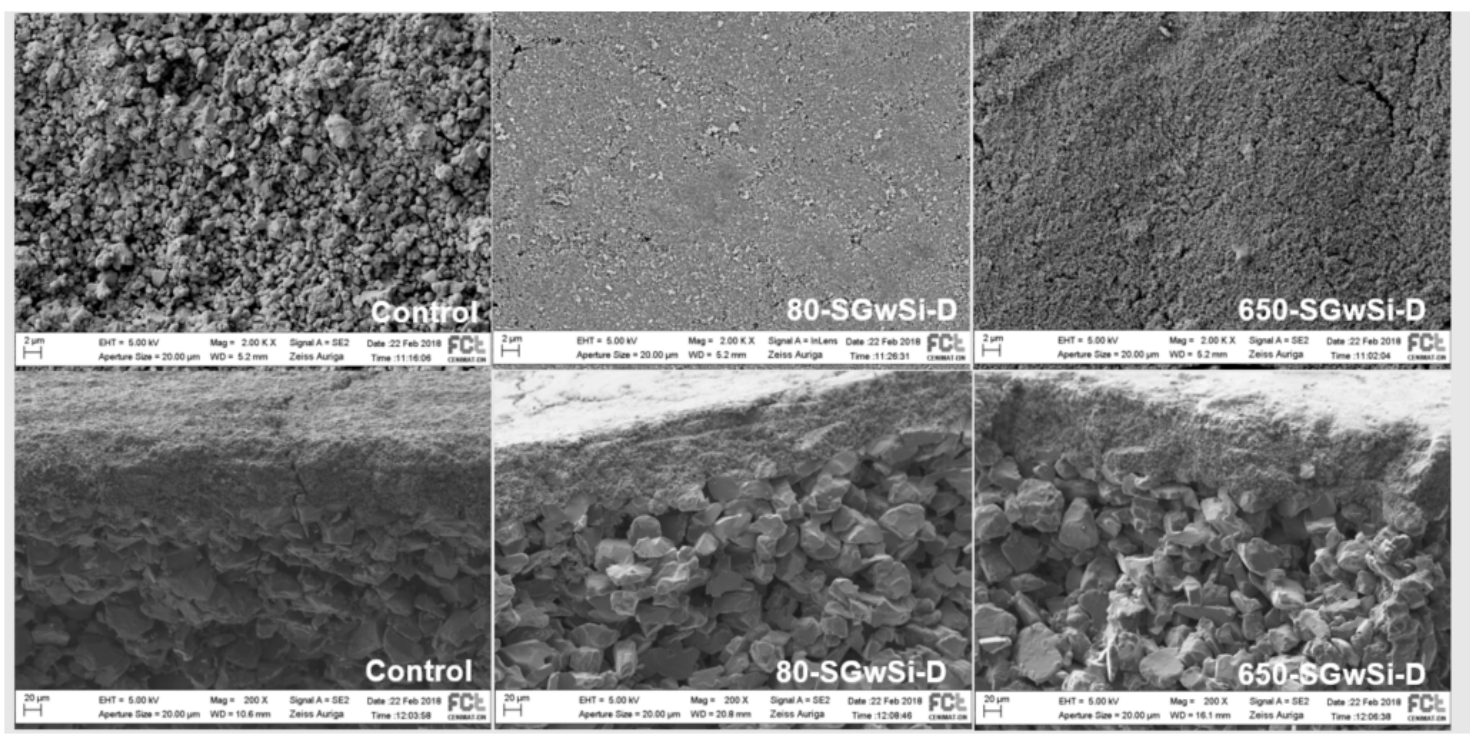

Figure 5. Top view (above) and cross-section (below) of the SEM images obtained for the control membranes, as well as for the solvent-free modified 80-SGwSi-D and 650-SGwSi-D membranes (magnification $\times 2000)$.

Porosity of Membranes

The porous characteristics of the membranes (80-SGwSi-D, 650-SGwSi-D, and control) were estimated in two different zones observed $\left(Z_{1}\right.$ and $\left.Z_{2}\right)$ after image analysis with the software ImageJ (Table 3). The zone selection was random. Two zones were analyzed to check if the membrane morphology changes after the modifications were consistent.

Table 3. Porous characterization of the membranes (magnification of $x 2000$ ).

\begin{tabular}{|c|c|c|c|c|c|c|}
\hline Membranes * & $\begin{array}{c}\text { Control } \\
\mathrm{Z}_{1} \times 2000\end{array}$ & $\begin{array}{c}\text { Control } \\
\mathrm{Z}_{2} \times \mathbf{2 0 0 0}\end{array}$ & $\begin{array}{c}80-S G w S i-D \\
Z_{1} \times 2000\end{array}$ & $\begin{array}{c}80-S G w S i-D \\
Z_{2} \times 2000\end{array}$ & $\begin{array}{c}\text { 650-SGwSi-D } \\
Z_{1} \times 2000\end{array}$ & $\begin{array}{c}\text { 650-SGwSi-D } \\
Z_{2} \times 2000\end{array}$ \\
\hline Pore density $\left(\mu \mathrm{m}^{-2}\right)$ & 1.7 & 1.5 & 11.6 & 11.1 & 3.8 & 10.1 \\
\hline Mean pore area $\left(\mu \mathrm{m}^{2}\right)$ & $0.05 \pm 0.16$ & $0.06 \pm 0.23$ & $0.01 \pm 0.02$ & $0.01 \pm 0.03$ & $0.01 \pm 0.03$ & $0.01 \pm 0.01$ \\
\hline Minimum pore area $\left(\mu \mathrm{m}^{2}\right)$ & 0.003 & 0.003 & 0.003 & 0.003 & 0.003 & 0.003 \\
\hline Maximum pore area $\left(\mu \mathrm{m}^{2}\right)$ & 3.409 & 7.136 & 0.79 & 1.00 & 2.15 & 0.19 \\
\hline Porosity (\%) & 9.0 & 9.3 & 12.0 & 8.6 & 3.4 & 6.3 \\
\hline Average circularity & $0.77 \pm 0.39$ & $0.80 \pm 0.26$ & $0.90 \pm 0.19$ & $0.93 \pm 0.16$ & $0.93 \pm 0.16$ & $0.94 \pm 0.15$ \\
\hline Average feret diameter $(\mu \mathrm{m})$ & $0.32 \pm 0.26$ & $0.33 \pm 0.45$ & $0.15 \pm 0.12$ & $0.13 \pm 0.11$ & $0.13 \pm 0.13$ & $0.12 \pm 0.07$ \\
\hline Maximum feret's diameter $(\mu \mathrm{m})$ & 1.000 & 6.963 & 3.215 & 2.896 & 5.461 & 1.079 \\
\hline Minimum feret's diameter $(\mu \mathrm{m})$ & 0.073 & 0.083 & 0.081 & 0.078 & 0.076 & 0.079 \\
\hline
\end{tabular}

The lower mean pore area obtained for the modified membranes (80-SGwSi-D and 650-SGwSi-D; $0.01 \mu \mathrm{m}^{2}$ ) compared to the unmodified membrane (control; $0.05 \mu \mathrm{m}^{2}$ ) might be indicative of a lower molecular weight cut off and might consequently lead to a higher pollutant rejection of the modified membranes (Table 3 ). Higher circularity values were determined for the modified membranes compared to the control, showing that the pores obtained were closer to perfect circles. The error bars associated with this parameter were also lower, showing a higher homogeneity for the modified 80-SGwSi-D and 650-SGwSi-D membranes compared to the control membrane.

Compared with the unmodified membrane (control), the modified membranes (80-SGwSi-D and 650-SGwSi-D) showed a much higher pore density (Table 3).

The average percent of porosity was slightly higher for the $80-\mathrm{SGwSi-D}$ membrane $(10.3 \%)$ compared to the control membrane $(9.1 \%)$. 
Feret's diameters of $0.32-0.33 \mu \mathrm{m}$ were observed for the control membrane, and, as expected, lower values of $0.12-0.15 \mu \mathrm{m}$ were observed for the two modified membranes (Table 3).

The modified membrane subject to $80^{\circ} \mathrm{C}$ (80-SGwSi-D) had a higher homogeneity (since a higher similarity was observed in the estimated parameters in two different zones), showing that this was the best modification to achieve a reproducible narrow pore size distribution and symmetry of pores.

\subsection{Contact Angle}

The hydrophilic properties of the membranes tested in terms of photocatalytic activity were determined by measuring the water contact angle using the sessile drop method.

Higher hydrophilicity for 80-SGwSi-D and 650-SGwSi-D membranes was obtained as expected due to the increase in $\mathrm{OH}$ groups presence induced by UV-Light [25], exhibiting values for the first contact angle of 11 and 14, respectively, whereas the control membrane achieved a first average contact angle of 34 degrees (Figure S2). Comparing the contact angle obtained for 80-SGwSi-D with a modified membrane reported in a previous study using ethanol as a solvent (named $\mathrm{SiO}_{2}-\mathrm{TiO}_{2} \mathrm{~L} 3$ ) [19], the average contact angle obtained was similar (11 degrees), and thus the use of a free solvent process was also beneficial for achieving high hydrophilic membranes and, potentially, lower fouling tendency.

\subsection{Membrane Filtration Performance}

The hydraulic permeability of the control and the solvent-free 80-SGwSi-D and 650-SGwSi-D membranes were measured using a dead-end filtration system previously described [19]. The control membrane presented a higher hydraulic permeability $\left(20,360 \pm 3583 \mathrm{Lh}^{-1} \mathrm{~m}^{-2} \mathrm{bar}^{-1}\right)$ when compared with the modified membranes (the membrane $80-\mathrm{SGwSi}-\mathrm{D}$ presented a hydraulic permeability of $4591 \pm 371 \mathrm{Lh}^{-1} \mathrm{~m}^{-2} \mathrm{bar}^{-1}$, and the membrane $650-\mathrm{SGwSi}-\mathrm{D}$ presented a hydraulic permeability of $\left.1864 \pm 472 \mathrm{Lh}^{-1} \mathrm{~m}^{-2} \mathrm{bar}^{-1}\right)$, which is a consequence of the higher pore size of the control membrane (Table 3). Table 4 shows the permeability for methylene blue during the filtration tests for the control and the solvent-free 80-SGwSi-D and 650-SGwSi-D membranes, measured in the absence and presence of UV radiation.

Table 4. Permeability, as well as methylene blue photocatalytic degradation and total removal during membrane filtration (MF), conducted with and without photolysis (UV).

\begin{tabular}{ccccccc}
\hline & \multicolumn{2}{c}{ Control } & \multicolumn{2}{c}{ 80-SGwSi-D } & \multicolumn{2}{c}{ 650-SGwSi-D } \\
\cline { 2 - 7 } & MF & MF + UV & MF & MF + UV & MF & MF + UV \\
\hline $\begin{array}{c}\text { MB permeability } \\
\left(\mathbf{L h}^{-1} \mathbf{m}^{-2} \text { bar }^{-1} \text { ) during }\right. \\
\text { the test }\end{array}$ & $19,126 \pm 950$ & $19,857 \pm 3490$ & $3479 \pm 280$ & $2645 \pm 705$ & $1955 \pm 135$ & $2486 \pm 363$ \\
\hline $\begin{array}{c}\text { Time of filtration (min) } \\
\text { \% Photocatalytic }\end{array}$ & 5 & 5 & 30 & 38 & 50 & 37 \\
\hline $\begin{array}{c}\text { degradation in the feed } \\
\text { \% Total removal }\end{array}$ & n.a & 0 & n.a & 31 & n.a & 39 \\
\hline
\end{tabular}

Figure 6 represents the evolution of methylene blue degradation in the feed and permeate streams as a function of the volume filtrated normalized by the membrane area that will allow the comparison of these results with others obtained using different membrane areas.

The results obtained in Figure 6 showed that the control membrane could not retain methylene blue despite the initial small decrease in the concentration measured in the permeate due to adsorption. This result was expected, given the small molecular weight of methylene blue, and concurred with a result previously reported [19]. For the 80-SGwSi-D and 650-SGwSi-D membranes, in the absence of light, a more accentuated initial decrease of the methylene blue concentration was observed in the permeate as a consequence of adsorption, more pronounced for the 650-SGwSi-D membrane. This result showed that the reduction of the pore size of the modified membranes was translated into a higher adsorption capacity. Adsorption was the reason assumed for the decrease of the concentration 
of methylene blue in the permeate samples since, as with time, the concentration of methylene blue in the permeate increased, tending to the concentration of the feed $(30 \mu \mathrm{M})$ in the assays conducted without UV.

The filtration tests conducted in the presence of UV light clearly showed the photocatalytic activity of both modified membranes. A clear decrease of methylene blue concentration was observed in the feed compartment in both tests due to its photodegradation, with a consequent downward trend of the concentration of methylene blue in the permeate in the case of the membrane 80-SGwSi-D, or achieving a constant concentration, in the case of the membrane 650-SGwSi-D.

Table 4 shows the significant improvement in the removal of methylene blue at the end of the filtration and UV experiments conducted with the modified membranes 80-SGwSi-D and 650-SGwSi-D to values of $37 \%$ and $45 \%$, respectively. Taking into account that the feed vessel was completely stirred and the permeate flux constant, the estimated average exposure time of methylene blue was $19 \mathrm{~min}$ for both membranes (Table 4).
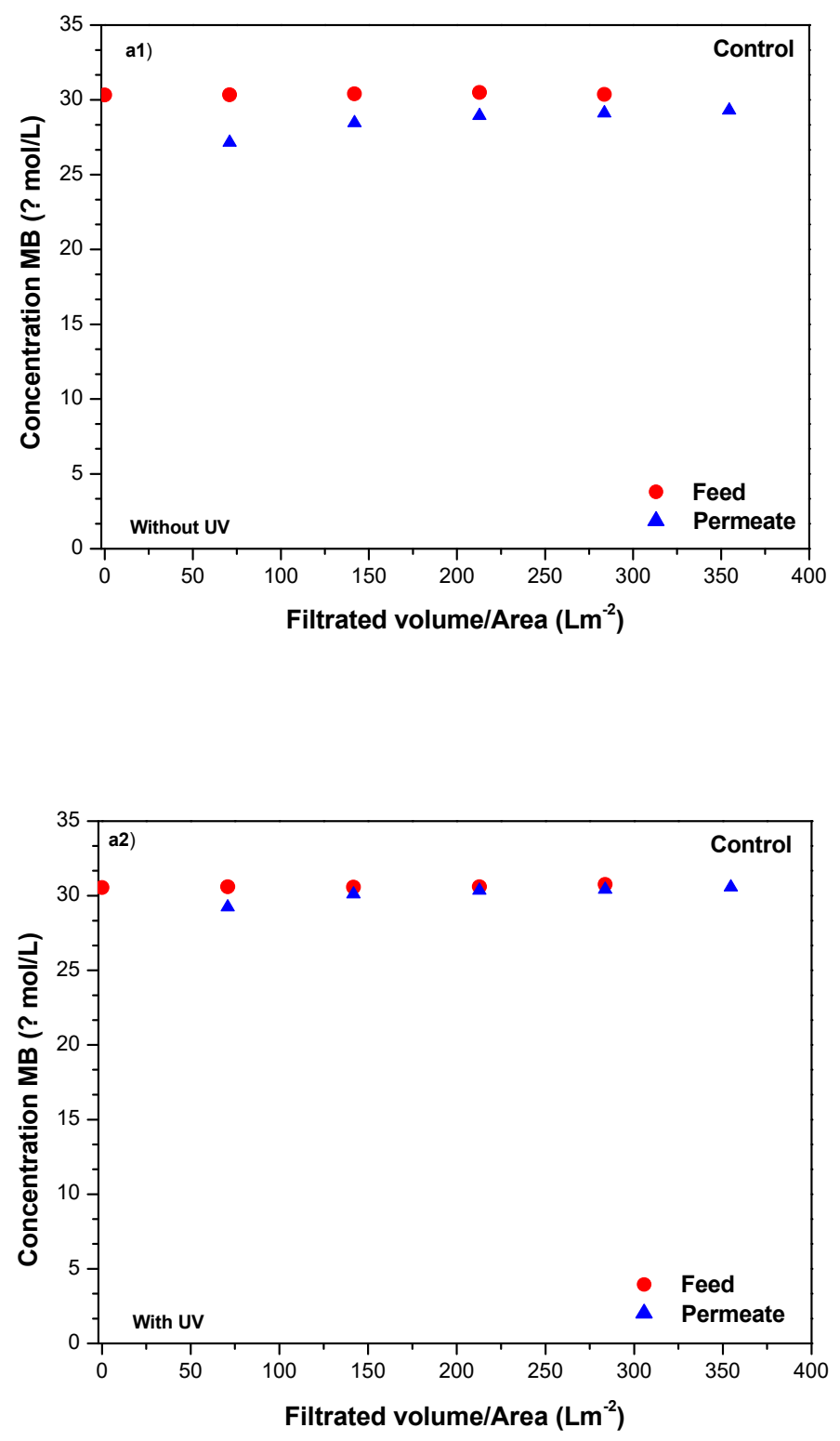

Figure 6. Cont. 

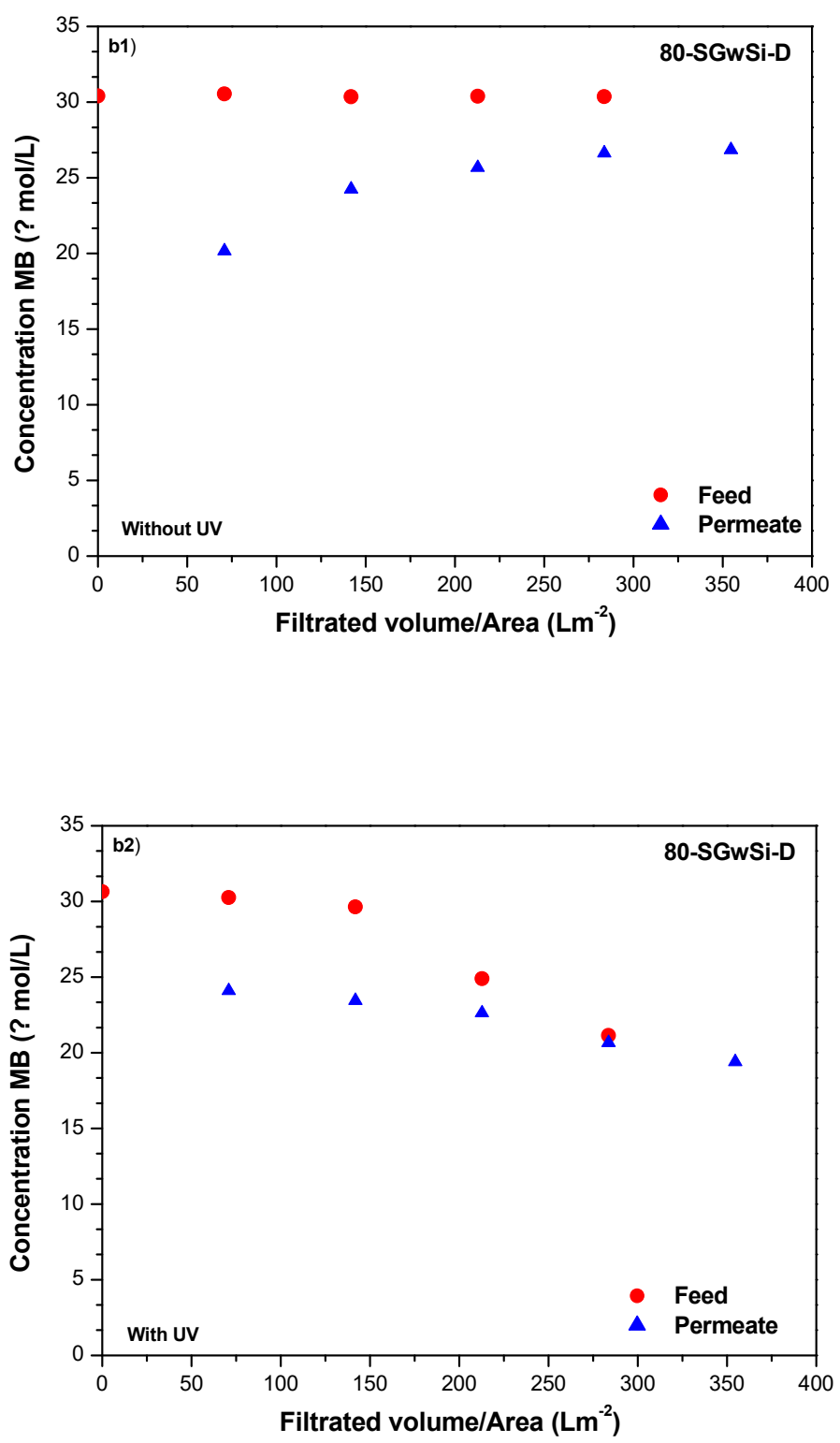

Figure 6. Cont. 

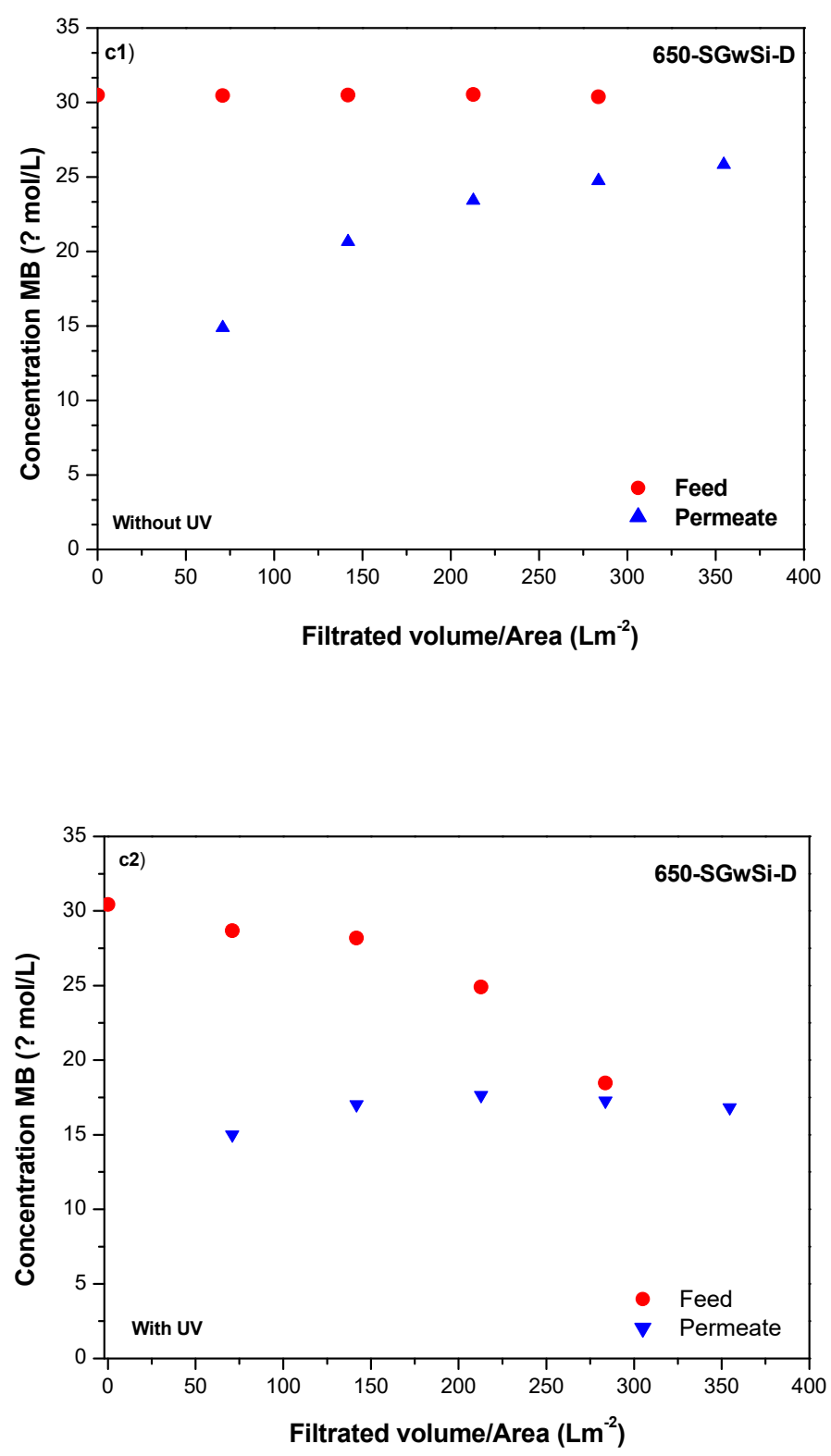

Figure 6. Comparison of filtration performance measured in terms of methylene blue (MB) concentration in feed and permeate samples with and without photolysis for the (a) control membrane, (b) modified 80-SGwSi-D membrane, and (c) the 650-SGwSi-D membrane.

The chemical stability of the modified membranes (80-SGwSi-D and 650-SGwSi-D) were also tested to ensure that the $\mathrm{TiO}_{2}$ coating was not released from the substrate, after cleaning with distilled water and acid and basic solutions [4]. The results obtained by inductively coupled plasma-atomic emission spectroscopy are presented in Table 5.

Results showed that the concentration of titanium in the solutions after the membrane cleaning experiments were similar to the concentrations measured in the control solutions, confirming the chemical stability of the modified membranes.

Given their characterization results (Table 3) and the results obtained in terms of photocatalytic performance (Figures 3 and 4), these membranes might prove effective in retaining microorganisms (such as bacteria and fungi) and inactivating them. Work is ongoing to prove these applications. As an example, when photocatalytic ceramic membranes, produced using this solvent-free method, were tested to treat surface water spiked with Aspergillus fumigatus, the results obtained showed high percentages of adsorption and retention of the spores for all treatments and that UV photolysis 
assured an effective treatment of the retentate [26]. In addition, the membranes produced in this work (without using solvents) proved to have a similar morphology and filtration performance when compared to photocatalytic membranes produced using solvents [19]. Since the photocatalytic membranes produced using solvents proved to be effective to treat wastewaters generated by the olive oil industry [20], the membranes detailed in this work are also expected to be effective to achieve high removals of total suspended solids, chemical oxygen demand, total organic carbon, and phenolic and volatile compounds.

Table 5. The concentration of titanium measured in control solutions and after filtration with three different cleaning solutions at $65 \pm 5{ }^{\circ} \mathrm{C}$ (distilled water, citric acid $2 \%(w / v)$ and $\mathrm{NaOH} 4 \%(w / v)$ using the control membrane and the modified 80-SGwSi-D and 650-SGwSi-D membranes.

\begin{tabular}{clc}
\hline \multirow{3}{*}{ Control solutions, no filtration performed } & \multicolumn{1}{c}{ Samples } & Ti (mg/L) \\
\cline { 2 - 3 } & Distilled water & $<0.005$ \\
\cline { 2 - 3 } Control membrane & Citric acid 2\% $(w / v)$ & 0.05 \\
\cline { 2 - 3 } & NaOH 4\% $(w / v)$ & 0.05 \\
\hline \multirow{2}{*}{ 80-SGwSi-D membrane } & Distilled water & 0.03 \\
\cline { 2 - 3 } & Citric acid 2\% $(w / v)$ & 0.04 \\
\cline { 2 - 3 } & NaOH 4\% $(w / v)$ & 0.09 \\
\cline { 2 - 3 } & Cistilled water & $<0.005$ \\
\cline { 2 - 3 } & Citric acid 2\% $(w / v)$ & 0.05 \\
\hline \multirow{2}{*}{ 650-SGwSi-D membrane } & NaOH 4\% $(w / v)$ & 0.05 \\
\hline & Distilled water & $<0.005$ \\
\cline { 2 - 3 } & Citric acid 2\% $(w / v)$ & 0.05 \\
\cline { 2 - 3 } & NaOH 4\% $(w / v)$ & 0.12 \\
\hline
\end{tabular}

\subsection{Economic Benefits of using Water Instead of Solvents}

The aim of this study was to adapt the production of $\mathrm{SiO}_{2}-\mathrm{TiO}_{2}$ sol-gel photocatalysts to modify commercial ceramic membranes rendering low cost and high productive photocatalytic materials for water decontamination.

Using water instead of an organic solvent in an industrial process brings environmental and economic benefits. The savings of ethanol achieved with this new method to produce photocatalytic membranes were calculated. The photocatalytic membrane (produced with ethanol) was already tested with real olive mill wastewaters with extremely promising results [20]. In this work, an average flux of $10 \mathrm{~L} /\left(\mathrm{m}^{2} \mathrm{~h}\right)$ was obtained at 0.2 bar with a membrane with $0.029 \mathrm{~m}^{2}$ of area.

Thus, a membrane area of $82 \mathrm{~m}^{2}$ would be needed to treat a total annual volume of $1000 \mathrm{~m}^{3}$ $\left(7 \mathrm{~m}^{3} /\right.$ day during five months, working $8 \mathrm{~h} /$ day) [27]. Given this, $1417 \mathrm{~L}$ of ethanol could be saved if these new solvent-free photocatalytic membranes were used. Thinking in economic terms, this represents a saving of $990 €$, considering an average cost of $0.7 € / \mathrm{L}$ of industrial ethanol and $1.55 € / \mathrm{m}^{3}$ of industrial water in Portugal.

\section{Materials and Methods}

In the present study, commercial flat sheet silicon carbide membranes were provided by LiqTech International and used as substrates. The chosen reagent for sol-gel preparation of the $\mathrm{SiO}_{2}$ precursor was tetraethyl orthosilicate (TEOS) (98\%; Sigma-Aldrich, St Louis, MO, USA). Degussa P25 titanium dioxide with 30-90 nm of nominal diameter provided by Evonik was used. All solvents employed in the sol-gel process were reagent-grade and used without further purification. Methylene blue (Merck, Darmstadt, Germany) was chosen to test the photocatalytic effectiveness of the modified 
membranes [28], and distilled water was used to measure the contact angle of the unmodified (control) and modified surfaces.

\subsection{Modification of Ceramic Membranes}

Silicon carbide flat membranes were cut (squares with an area of $11.4 \mathrm{~cm}^{2}$ used in the assays to test the photocatalytic performance detailed in Section 3.2.1, and circles with an area of $17.3 \mathrm{~cm}^{2}$ used in the membrane filtration tests detailed in Section 3.2.4; thickness of $0.3 \mathrm{~cm}$ ), thoroughly cleaned with a $2 \%$ solution of MicroClean $90^{\circledR}$, rinsed with distilled water, and heated at $80{ }^{\circ} \mathrm{C}$ overnight. Drop-casting was used as a deposition method. A previous modification strategy that proved to be effective in terms of photocatalytic activity, but employed solvents, served as the basis for this work [19]. The strategy was to use silicon carbide substrates and modify them using TEOS as silicon dioxide source, combined with Degussa nanoparticles as titania source. The optimized procedure was tested following a solvent-free sol-gel procedure conducted at room temperature conditions.

\subsubsection{Temperature Effect on Photocatalytic Activity}

The membranes were coated with three layers of silicon dioxide combined with titanium dioxide Degussa (D) P25 nanoparticles (Figure 7), as detailed in previous work using ethanol as a solvent under acid-catalyzed sol-gel synthesis [19]. To test the influence of the final thermal protocol on the photocatalytic behavior of the membranes, the modified membranes were subject (at ambient atmosphere) to ramps of slow temperature increase $\left(3{ }^{\circ} \mathrm{C} / \mathrm{min}\right)$, up to $300{ }^{\circ} \mathrm{C}, 500{ }^{\circ} \mathrm{C}$, and $650{ }^{\circ} \mathrm{C}$. These temperatures were selected since the range $500{ }^{\circ} \mathrm{C}$ to $600^{\circ} \mathrm{C}$ has been described as optimal for achieving good final photocatalytic properties in $\mathrm{SiO}_{2}-\mathrm{TiO}_{2}$ compositions [29] while ensuring the thermal stability of the commercial silicon carbide substrate. High temperatures (e.g., above $800{ }^{\circ} \mathrm{C}$ ) may induce thermal stress by inducing defects at the $\mathrm{SiO}_{2} / \mathrm{SiC}$ interface [30]. The selected temperatures $\left(300^{\circ} \mathrm{C}, 500{ }^{\circ} \mathrm{C}\right.$, and $\left.650^{\circ} \mathrm{C}\right)$ were maintained for three hours, and the samples were then cooled down naturally. The membranes obtained were labelled as T-SGSi-D, where T denotes the temperature applied.

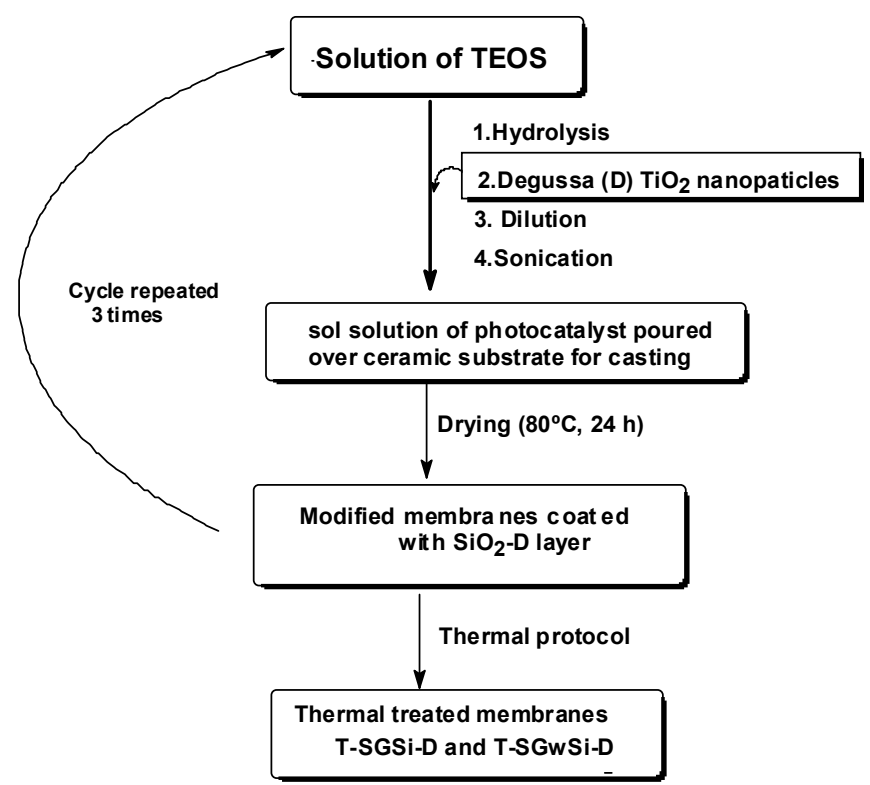

Figure 7. Representation of the modification process and designation of the membranes produced. $\mathrm{T}$ denotes the temperature applied over modified substrates samples and $\mathrm{w}$ differentiates the samples prepared using solvent free sol-gel process. 


\subsubsection{Solvent-Free Process for the Production of Photocatalytic Membranes}

The silicon carbide substrates were modified using the sol-gel process described above [19] but employing an aqueous matrix, maintaining the concentration of the acid catalyst, TEOS, and Degussa nanoparticles (Figure 7).

To avoid using ethanol, in this work, a concentrated stock solution of TEOS (1.69 M) was prepared in aqueous acidic conditions $(\mathrm{pH}=1)$. The hydrolysis and condensation reactions to convert the silicon alkoxide TEOS, completely to $\mathrm{SiO}_{2}$, was guaranteed because two moles of water are needed for each mole of Si precursor. So, an excess of water can be used [31]. TEOS reagent is not miscible in water, so it is typically mixed with ethanol to improve its dispersion in water [32]. However, in this work, the miscibility was attained after $20 \mathrm{~min}$ due to the addition of acid as catalyst. The hydrolysis was maintained during $2 \mathrm{~h}$, and the transparent stock solution was stable for one month. The hydrolyzed TEOS was diluted with distilled water and added to Degussa nanoparticles, with a final concentration of $0.045 \mathrm{M} \mathrm{SiO}_{2}$ and $0.050 \mathrm{M} \mathrm{TiO}_{2}$ that corresponds to 0.9:1 molar ratio of $\mathrm{SiO}_{2}: \mathrm{TiO}_{2}[16,19]$. One milliliter of this sonicated sol-solution was deposited over silicon carbide membranes $\left(11.4 \mathrm{~cm}^{2}\right)$ by drop-casting and heated at $80^{\circ} \mathrm{C}$ for $24 \mathrm{~h}$ to promote the condensation and form the $\mathrm{SiO}_{2}$ network. This protocol was repeated three times. A weight of $12 \mathrm{mg} \mathrm{TiO}{ }_{2}$ was, therefore, coated in each membrane piece with $11.4 \mathrm{~cm}^{2}\left(1 \mathrm{mg} / \mathrm{cm}^{2}\right)$. For the circular membranes used in the filtration tests, a weight of $18.3 \mathrm{mg} \mathrm{TiO}$ was coated in each membrane piece with $17.3 \mathrm{~cm}^{2}$ to achieve the same value of weight per unit area of $1 \mathrm{mg} / \mathrm{cm}^{2}$.

The membranes modified by this procedure were compared to membranes subjected to an additional thermal protocol: ramp of $3{ }^{\circ} \mathrm{C} / \mathrm{min}$ up to a maximum temperature of $650^{\circ} \mathrm{C}$ maintained during $3 \mathrm{~h}$ (at ambient atmosphere) and cooled down naturally.

The modified membranes obtained were identified as T-SGwSi-D, where T denotes the temperature applied over modified substrates samples, and $\mathrm{w}$ differentiates the samples prepared using water as a matrix in the sol-gel process.

\subsection{Assessment of the Photocatalytic Membranes and Selection of the Most Promising One}

\subsubsection{Assays to Test the Photocatalytic Performance}

The evaluation of the photocatalytic activity of the modified membranes was performed, as previously described [19]. Methylene blue was employed as a tester dye to test the photocatalytic efficiency of the unmodified (control) and modified membranes [28]. A UV collimated beam set-up with an UVH-lamp type Z (UV-Technik meyer GmbH, Ortenberg, Germany) that emits polychromatic light, housed in a shuttered box with PN310 quartz (UV-Technik), was used. A calibrated radiometer (IL393, International Light, Newburyport, MA, USA), placed at the same height of the solution level in the Petri dish was used, to measure the maximum irradiance value $\left(25 \mathrm{~mW} / \mathrm{cm}^{2}\right)$.

Double-walled glass Petri dishes (maintained at $23 \pm 2{ }^{\circ} \mathrm{C}$ by the circulation of cold water) were placed beneath the UV source with $30 \mathrm{~mL}$ of a constantly stirred aqueous solution of $30 \mu \mathrm{M}$ of methylene blue ( $\mathrm{pH}$ 6.3) with and without a membrane (to test the direct photolysis effect). The concentration of methylene blue was measured in the different samples taken at different experimental times $(0,20,40$, and $60 \mathrm{~min}$ ) and quantified based on a calibration curve performed after measuring the absorbance measurements at $664 \mathrm{~nm}$ using a UV-Vis spectrophotometer (Ultrospec 2100 pro-UV-VIS, Biochrom Ltd., Cambridge, UK). Four absorbance measurements were taken in each sample. To test the adsorption capacity of the unmodified and modified membranes, dark reactions were performed under the same conditions. The percent removal obtained after $60 \mathrm{~min}$ was calculated using Equation (2):

$$
\% \text { removal of methylene blue }=\left[\frac{\left(C_{0}-C_{60}\right)}{C_{0}}\right] \times 100
$$


where $C_{0}$ is the concentration of the methylene blue measured at time zero and $C_{60}$ the concentration measured after 60 min of UV exposure.

For the most promising membranes, five consecutive degradation experiments were conducted under the same conditions using two different batches of membranes modified to ensure the photocatalytic results obtained were reproducible and that the membranes could be reused.

A last long-term assay was conducted with the most promising membranes to follow the degradation of methylene blue.

Membrane characterization (in terms of morphology and contact angle) was performed after membrane evaluation in terms of their photocatalytic activity.

\subsubsection{Morphology Characterization}

Scanning electron microscopy (SEM) was used to characterize the top surface and cross-section of the most promising modified membranes and compare it with the unmodified substrate after coating with an $\mathrm{Au} / \mathrm{Pd}$ thin film $(15 \mathrm{~nm})$. They were analyzed using a Carl Zeiss AURIGA CrossBeam Workstation instrument equipped with an Oxford Energy Dispersive X-ray Spectrometer (EDS). The different top surfaces were analyzed using the ImageJ software (an open source image processing program, University of Wisconsin, Madison, WI, USA) [33-35]. Two random zones were analyzed to check if the membrane morphology was consistent.

\subsubsection{Contact Angle}

Three different places were randomly chosen in each membrane to measure the contact angle of a sessile drop of distilled water (10-12 $\mu \mathrm{L})$ using a KSV CAM2008 equipment. Twenty frames were attained for each measurement with a frame interval of $100 \mathrm{~ms}$.

\subsubsection{Membrane Filtration Assays}

A dead-end filtration system coupled to a vacuum pump (model: DOA-P504A-BN, GAST Manufacturing) was placed under the UV system to compare the performance of the different solvent-free modified membranes (80-SGwSi-D and 650-SGwSi-D) produced in this work and the control. A total of $250 \mathrm{~mL}$ of a $30 \mu \mathrm{M}$ methylene blue solution was filtered by the $4.7 \mathrm{~cm}$ diameter membranes with a 0.2 bar transmembrane pressure using this setup. Control assays were conducted in the absence of UV light. The total removal of methylene blue that includes the effect of membrane filtration (rejection and adsorption) and photolysis (direct and indirect photodegradation) was calculated using Equation (3):

$$
\% \text { total removal of methylene blue }=\left[\frac{\left(C_{\text {feed }}-C_{\text {permeate }}\right)}{C_{\text {feed }}}\right] \times 100
$$

The photocatalytic degradation of methylene blue in the feed compartment was calculated through Equation (4):

$$
\% \text { photocatalytic degradation of methylene blue }=\frac{\left(C_{\text {feed }_{t}}-C_{\text {feed }_{t-1}}\right)}{C_{\text {feed }_{t}}}
$$

After the filtration experiments, different cleaning protocols, described by Fraga et al. [4] in pilot-scale experiments conducted with tubular $\mathrm{SiC}$ unmodified membranes, were tested to assess the chemical resistance of the modified membranes used in the experiments described above by monitoring the possible release of nanomaterials from the membranes. The cleaning protocol consisted of rinsing the membrane with $100 \mathrm{~mL}$ of water, followed by chemical cleanings using $100 \mathrm{~mL}$ of $\mathrm{NaOH} 4 \%(w / v)$ and citric acid $2 \%(w / v)$ solutions. All the steps were performed at $65 \pm 5^{\circ} \mathrm{C}$ [4]. The Ti element was 
analyzed by inductively coupled plasma-atomic emission spectroscopy (ICP-AES) (Horiba Jobin-Yvon, Longjumeau, France).

\section{Conclusions}

A new solvent-free sol-gel process was proposed to produce photocatalytic membranes that could be reused and maintain their photocatalytic activity. The modification process proposed was low cost and environmentally friendly (without the use of solvents and involving a low-temperature thermal treatment). These membranes proved to be effective and might be promising for the removal and inactivation of microorganisms from wastewater. Therefore, these membranes should be further tested in pilot-scale hybrid reactors that combine membrane filtration, $\mathrm{UV}$, and advanced oxidation processes.

Supplementary Materials: The following are available online, Figure S1: Pseudo-first-order kinetics for the removal of methylene blue for membranes tested; Figure S2: Comparison of the time course of water contact angle for control, 80-SGwSi-D, and 650-SGwSi-D modified substrates.

Author Contributions: All authors contributed to the design and concept of this article. Conceptualization, R.M.H., V.J.P., and J.G.C; Methodology, R.M.H. and M.C.F.; Investigation, V.J.P., R.M.H., and M.C.F.; Writing-Original Draft Preparation, R.M.H; Writing-Review and Editing, R.M.H, V.J.P., M.C.F., and J.G.C.; Visualization, V.J.P.; Supervision, V.J.P. and J.G.C.

Funding: Financial support from Fundação para a Ciência e a Tecnologia-through the project PTDC/EAM-AMB/30989/2017 financed by the state budget is gratefully acknowledged.

Acknowledgments: The authors acknowledge Elvira Fortunato and Daniela Nunes, from i3N/CENIMAT, Department of Materials Science, Faculty of Science and Technology, Universidade NOVA de Lisboa, for the SEM images. iNOVA4Health - UID/Multi/04462/2013, a program financially supported by Fundação para a Ciência e Tecnologia/Ministério da Educação e Ciência, through national funds and co-funded by FEDER under the PT2020 Partnership Agreement is gratefully acknowledged. Funding from INTERFACE Program, through the Innovation, Technology, and Circular Economy Fund (FITEC), is gratefully acknowledged. Associate Laboratory for Green Chemistry LAQV, which is also financed by national funds from FCT/MEC (UID/QUI/50006/2013) and co-financed by the ERDF under the PT2020 Partnership Agreement (POCI-01-0145-FEDER - 007265), is gratefully acknowledged.

Conflicts of Interest: The authors declare no conflict of interest. The funders had no role in the design of the study; in the collection, analyses, or interpretation of data; in the writing of the manuscript, or in the decision to publish the results.

\section{References}

1. Liu, H.; Li, C.; Ren, X.; Liu, K.; Yang, J. Fine platinum nanoparticles supported on a porous ceramic membrane as efficient catalysts for the removal of benzene. Sci. Rep. 2017, 7, 16589. [CrossRef] [PubMed]

2. Antolini, E.; Gonzalez, E.R. Ceramic materials as supports for low-temperature fuel cell catalysts. Solid State Ion. 2009, 180, 746-763. [CrossRef]

3. Zhou, Y.; Fukushima, M.; Miyazaki, H.; Yoshizawa, Y.I.; Hirao, K.; Iwamoto, Y.; Sato, K. Preparation and characterization of tubular porous silicon carbide membrane supports. J. Memb. Sci. 2011, 369, 112-118. [CrossRef]

4. Fraga, M.C.; Sanches, S.; Crespo, J.G.; Pereira, V.J. Assessment of a new silicon carbide tubular honeycomb membrane for treatment of olive mill wastewaters. Membranes 2017, 7, 12. [CrossRef]

5. Vaccaro, L.; Lanari, D.; Marrocchi, A.; Strappaveccia, G. Flow approaches towards sustainability. Green Chem. 2014, 16, 3680-3704. [CrossRef]

6. Fraga, M.C.; Sanches, S.; Pereira, V.J.; Crespo, J.G.; Yuan, L.; Marcher, J.; de Yuso, M.V.M.; Rodríguez-Castellón, E.; Benavente, J. Morphological, chemical surface and filtration characterization of a new silicon carbide membrane. J. Eur. Ceram. Soc. 2017, 37, 899-905. [CrossRef]

7. Mozia, S. Photocatalytic membrane reactors (PMRs) in water and wastewater treatment. A review. Sep. Purif. Technol. 2010, 73, 71-91. [CrossRef]

8. Sanches, S.; Nunes, C.; Passarinho, P.C.; Ferreira, F.C.; Pereira, V.J.; Crespo, J.G. Development of photocatalytic titanium dioxide membranes for degradation of recalcitrant compounds. J. Chem. Technol. Biot. 2016, 92, 1727-1737. [CrossRef] 
9. Geltmeyer, J.; Teixido, H.; Meire, M.; Van Acker, T.; Deventer, K.; Vanhaecke, F.; Van Hulle, S.; De Buysser, K.; De Clerck, $\mathrm{K}_{\text {. TiO }}$ functionalized nanofibrous membranes for removal of organic (micro) pollutants from water. Sep. Purif. Technol. 2017, 179, 533-541. [CrossRef]

10. Sun, L.; Wan, S.; Yu, Z.; Wang, L. Optimization and modeling of preparation conditions of $\mathrm{TiO}_{2}$ nanoparticles coated on hollow glass microspheres using response surface methodology. Sep. Purif. Technol. 2014, 125, 156-162. [CrossRef]

11. Rajoriya, S.; Bargole, S.; George, S.; Saharan, V.K.; Gogate, P.R.; Pandit, A.B. Synthesis and characterization of samarium and nitrogen doped $\mathrm{TiO}_{2}$ photocatalysts for photo-degradation of 4-acetamidophenol in combination with hydrodynamic and acoustic cavitation. Sep. Purif. Technol. 2019, 209, 254-269. [CrossRef]

12. Kim, J.; Van der Bruggen, B. The use of nanoparticles in polymeric and ceramic membrane structures: Review of manufacturing procedures and performance improvement for water treatment. Environ. Pollut. 2010, 158, 2335-2349. [CrossRef] [PubMed]

13. Brinker, C.J.; Sehgal, R.; Hietala, S.L.; Deshpande, R.; Smith, D.M.; Loy, D.; Ashley, C.S. Sol-gel strategies for controlled porosity inorganic materials. J. Membr. Sci. 1994, 94, 85-102. [CrossRef]

14. Langlet, M.; Kim, A.; Audier, M.; Herrmann, J.M. Sol-Gel preparation of photocatalytic $\mathrm{TiO}_{2}$ films on polymer substrates. J. Sol.-Gel Sci. Technol. 2002, 25, 223-234. [CrossRef]

15. Sun, L.; An, T.; Wan, S.; Li, G.; Bao, N.; Hu, X.; Fu, J.; Sheng, G. Effect of synthesis conditions on photocatalytic activities of nanoparticulate $\mathrm{TiO}_{2}$ thin films. Sep. Purif. Technol. 2009, 68, 83-89. [CrossRef]

16. Fateh, R.; Dillert, R.; Bahnemann, D. Preparation and characterization of transparent hydrophilic photocatalytic $\mathrm{TiO}_{2} / \mathrm{SiO}_{2}$ thin films on polycarbonate. Langmuir 2013, 29, 3730-3739. [CrossRef]

17. Gao, X.; Wachs, I.E. Titania-silica as catalysts: Molecular structural characteristics and physico-chemical properties. Catal. Today 1999, 51, 233-254. [CrossRef]

18. Kim, J.Y.; Jang, J.-W.; Youn, D.H.; Kim, E.S.; Choi, S.H.; Shin, T.J.; Lee, J.S. Photocatalytic selective oxidation of the terminal methyl group of dodecane with molecular oxygen over atomically dispersed Ti in a mesoporous $\mathrm{SiO}_{2}$ matrix. Green Chem. 2013, 15, 3387-3395. [CrossRef]

19. Huertas, R.M.; Fraga, M.C.; Crespo, J.G.; Pereira, V.J. Sol-gel membrane modification for enhanced photocatalytic activity. Sep. Purif. Technol. 2017, 180, 69-81. [CrossRef]

20. Fraga, C.; Huertas, R.; Crespo, J.G.; Pereira, V.J. Novel submerged photocatalytic membrane reactor for treatment of olive mill wastewaters. Catalysts 2019, 9, 769. [CrossRef]

21. DeVierno Kreuder, A.; House-Knight, T.; Whitford, J.; Ponnusamy, E.; Miller, P.; Jesse, N.; Rodenborn, R.; Sayag, S.; Gebel, M.; Aped, I.; et al. A method for assessing greener alternatives between chemical products following the 12 principles of green chemistry. ACS Sustain. Chem. Eng. 2017, 5, 2927-2935. [CrossRef]

22. Zhou, X.; Li, X.; Gao, Q.; Yuan, J.; Wen, J.; Fang, Y.; Liu, W.; Zhang, S.; Liu, Y. Metal-free carbon nanotube-SiC nanowire heterostructures with enhanced photocatalytic $\mathrm{H}_{2}$ evolution under visible light irradiation. Catal. Sci. Technol. 2015, 5, 2798-2806. [CrossRef]

23. Chen, Y.; Dionysiou, D.D. Correlation of structural properties and film thickness to photocatalytic activity of thick $\mathrm{TiO}_{2}$ films coated on stainless steel. Appl. Catal. B 2006, 69, 24-33. [CrossRef]

24. Wang, X.; Wu, G.; Zhou, B.; Shen, J. thermal annealing effect on optical properties of binary $\mathrm{TiO}(2)-\mathrm{SiO}(2)$ sol-gel coatings. Materials 2013, 6, 76-84. [CrossRef]

25. Cheng, Z.; Cheng, K.; Weng, W. $\mathrm{SiO}_{2} / \mathrm{TiO}_{2}$ Nanocomposite films on polystyrene for light-induced cell detachment application. ACS Appl. Mater. Interfaces 2017, 9, 2130-2137.

26. Oliveira, B.R.; Sanches, S.; Huertas, R.; Crespo, M.T.B.; Pereira, V.J. Treatment of a real water matrix inoculated with Aspergillus fumigatus using a photocatalytic membrane reactor. 2019. Submitted.

27. Sanches, S.; Fraga, M.C.; Silva, N.A.; Nunes, P.; Crespo, J.G.; Pereira, V.J. Pilot scale nanofiltration treatment of olive mill wastewater: A technical and economical evaluation. Environ. Sci. Pollut. Res. 2017, 24, 3506-3518. [CrossRef]

28. Mills, A. An overview of the methylene blue ISO test for assessing the activities of photocatalytic films. Appl. Catal. B 2012, 128, 144-149. [CrossRef]

29. Mahyar, A.; Behnajady Mohammad, A.; Modirshahla, N. Enhanced Photocatalytic degradation of c.i. basic violet 2 using $\mathrm{TiO}_{2}-\mathrm{SiO}_{2}$ composite nanoparticles. Photochem. Photobiol. Sci. 2011, 87, 795-801. [CrossRef]

30. Kim, D.-K.; Jeong, K.-S.; Kang, Y.-S.; Kang, H.-K.; Cho, S.W.; Kim, S.-O.; Suh, D.; Kim, S.; Cho, M.-H. Controlling the defects and transition layer in $\mathrm{SiO}_{2}$ films grown on $4 \mathrm{H}-\mathrm{SiC}$ via direct plasma-assisted oxidation. Sci. Rep. 2016, 6, 34945. [CrossRef] 
31. Fardad, M.A.; Yeatman, E.M.; Dawnay, E.J.C.; Green, M.; Horowitz, F. Effects of $\mathrm{H}_{2} \mathrm{O}$ on structure of acid-catalysed $\mathrm{SiO}_{2}$ sol-gel films. J. Non-Cryst. Solids 1995, 183, 260-267. [CrossRef]

32. Chen, H.-S.; Huang, S.-H.; Perng, T.-P. Preparation and characterization of molecularly homogeneous silica-titania film by sol-gel process with different synthetic strategies. Appl. Mater. Interfaces 2012, 4, 5188-5195. [CrossRef]

33. Almarzooqi, F.; Bilad, M.; Mansoor, B.; Arafat, H. A comparative study of image analysis and porometry techniques for characterization of porous membranes. J. Mater. Sci. 2016, 51, 2017-2032. [CrossRef]

34. Masselin, I.; Durand-Bourlier, L.; Laine, J.-M.; Sizaret, P.-Y.; Chasseray, X.; Lemordant, D. Membrane characterization using microscopic image analysis. J. Membrane Sci. 2001, 186, 85-96. [CrossRef]

35. Abramoff, M.D.; Magalhaes, P.J.; Ram, S.J. Image Processing with ImageJ. J. Biophotonics 2004, 11, 36-42.

Sample Availability: Samples are not available from the authors.

(C) 2019 by the authors. Licensee MDPI, Basel, Switzerland. This article is an open access article distributed under the terms and conditions of the Creative Commons Attribution (CC BY) license (http://creativecommons.org/licenses/by/4.0/). 